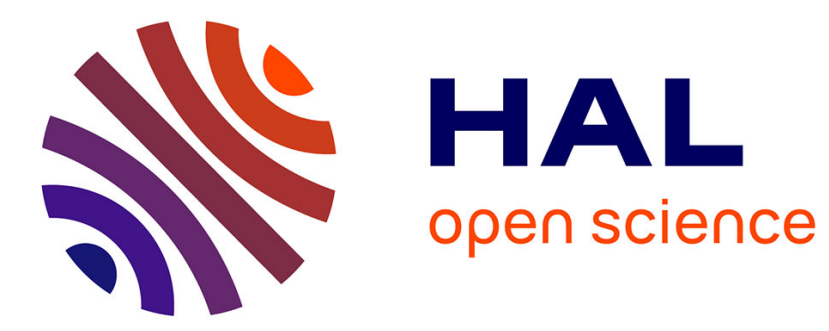

\title{
GeodesicSlicer: a Slicer Toolbox for Targeting Brain Stimulation
}

F. Briend, E. Leroux, C. Nathou, N. Delcroix, S. Dollfus, Olivier M Etard

\section{To cite this version:}

F. Briend, E. Leroux, C. Nathou, N. Delcroix, S. Dollfus, et al.. GeodesicSlicer: a Slicer Toolbox for Targeting Brain Stimulation. Neuroinformatics, 2020, 18 (4), pp.509-516. 10.1007/s12021-020-094579. hal-03047844

HAL Id: hal-03047844

https://hal-normandie-univ.archives-ouvertes.fr/hal-03047844

Submitted on 14 Dec 2020

HAL is a multi-disciplinary open access archive for the deposit and dissemination of scientific research documents, whether they are published or not. The documents may come from teaching and research institutions in France or abroad, or from public or private research centers.
L'archive ouverte pluridisciplinaire HAL, est destinée au dépôt et à la diffusion de documents scientifiques de niveau recherche, publiés ou non, émanant des établissements d'enseignement et de recherche français ou étrangers, des laboratoires publics ou privés. 


\section{Title Page}

\section{GeodesicSlicer: A Slicer toolbox for targeting brain stimulation}

Briend F. ${ }^{a^{*}}$, Leroux E. ${ }^{a}$, Nathou $C .{ }^{a, b}$, Delcroix N. ${ }^{c}$, Dollfus $S .{ }^{a, b}$, Etard O. ${ }^{a d}$.

${ }^{a}$ Normandie Univ, UNICAEN, ISTS, EA 7466, GIP Cyceron, 14000 Caen, France

${ }^{b}$ CHU de Caen, Service de Psychiatrie adulte, Centre Esquirol, 14000 Caen, France

${ }^{c}$ Normandie Univ, UNICAEN, CNRS, CHU de Caen, UMS 3408, GIP Cyceron, 14000 Caen,

France

${ }^{d}$ CHU de Caen, Service d'Explorations Fonctionnelles du Système Nerveux, 14000 Caen, France

* Corresponding author : Frédéric Briend, Centre Hospitalier Universitaire, Centre Esquirol, Caen, F-14000, France. Tel.: +33 231065018; fax: +33 231064987.

E-mail : $\underline{\text { briend@cyceron.fr. http://www.ists.cyceron.fr/ }}$

Abstract: 196 words

Text: 3596 words

Number of figures: 2 figures

Number of tables: 0 table 


\section{ABSTRACT}

NonInvasive Brain Stimulation (NIBS) is a potential therapeutic tool with growing interest, but neuronavigation-guided software and tools available for the target determination are mostly either expensive or closed proprietary applications. To address these limitations, we propose GeodesicSlicer, a customizable, free, and open-source NIBS therapy research toolkit. GeodesicSlicer is implemented as an extension for the widely used 3D Slicer medical image visualization and analysis application platform. GeodesicSlicer uses cortical stimulation target from either functional or anatomical images to provide functionality specifically designed for NIBS therapy research. The provided algorithms are tested and they are accessible through a convenient graphical user interface. Modules have been created for NIBS target determination according to the position of the electrodes in the 10-20 system electroencephalogram and calculating correction factors to adjust the repetitive Transcranial Magnetic Stimulation (rTMS) dose for the treatment. Two illustrative examples are processing with the module. This new open-source software has been developed for NIBS therapy: GeodesicSlicer is an alternative for laboratories that do not have access to neuronavigation system. The triangulation-based MRI-guided method presented here provides a reproducible and inexpensive way to position the TMS coil that may be used without the use of a neuronavigation system.

\section{Keywords}

NIBS, rTMS, 3D Slicer, EEG, target determination, correction factor. 


\section{Introduction}

A key issue in the field of NonInvasive Brain Stimulation (NIBS) is to determine an accurate localization on the scalp to correctly target cortical areas knowing the great anatomical variability of the brain. Since personalized medicine for the treatment of psychosis allows for the consideration of substantial inter-individual variability, recent findings claim that brain stimulation can be guided in a personalized manner (Briend et al. Under Review; Kraus and Gharabaghi 2015; Lahti 2016; I. E. Sommer et al. 2018).

Most clinical applications of the NIBS are based on probabilistic targeting methods which do not account for individual anatomical variability (e.g. for major depressive episodes the so called "5-cm rule" (U. Herwig et al. 2001) or the International 10-20 electroencephalogram (EEG) (De Witte et al. 2018; Uwe Herwig et al. 2003) or derivative system (Beam et al. 2009)). This may lead to suboptimal clinical responses when compared to individualized targeting techniques based on structural brain scanning. Research and clinical studies require accuracy and precision not offered by these probabilistic targeting methods (Herbsman and Nahas 2011). For example, a common and easy method for the positioning of the coil in psychiatric therapies uses the standardized T3P3 site according to the International 10-20 system of EEG electrode positioning (Jasper 1958). However, this method is known to be an inaccurate estimation, especially given its variable projections on the individual brain (Briend et al. Under Review; Uwe Herwig et al. 2003). It is why, there is a need for personalized target method that uses the participant's own anatomical or functional images to guide target placement.

As a personalized target method, the combination of brain imaging and a neuronavigation system in the field of NIBS may improve the efficacy of stimulation treatment (U. Herwig et al. 2001; I. E. Sommer et al. 2018; I. E. C. Sommer et al. 2007), however, there are some disadvantages. These include the high cost of these systems, which can exceed 
$\$ 50,000$, the complexity to use, the space consumed by the device and the difficulty in using these systems for the study of posterior brain areas located in the blind spot of the neuronavigation system (Vaghefi et al. 2015).

In order to propose an alternative that can combine the accuracy and the simplicity of the two previous methods, we developed an open-source tool "GeodesicSlicer", which facilitates the stimulation site determination, allowing users to manually posit the target of repetitive Transcranial Magnetic Stimulation (rTMS) coil over a cortical target derived from functional or anatomical images. This module creates a 3D mesh morphed to the structural MRI head data of the participant then projected an individualized 10-20 system EEG and the cortical stimulation target on it. Then, the module calculates the geodesic distances between the projected stimulation target and the position of the 3 nearest electrodes in the individualized 10-20 system EEG in order to guide the stimulation. Our technique takes triangulation-based MRI-guided method as Andoh et al. (Andoh et al. 2009) that devised a method of targeting NIBS using an anatomical scan only. Moreover, it was proposed that rTMS inter-individual variability in its efficacy for treating patient could be attributed to variations in the cortical anatomy in Schizophrenia (Ralph E. Hoffman et al. 2013) or in major depression disorders (MDD) (Trojak et al. 2012). It is why, we implemented in this module, correction factors, according to scalp-to-cortex distance (Summers and Hanlon 2017), to adjust the rTMS dose for the treatment.

We propose GeodesicSlicer as a common easy-to-use software for NIBS site determination, thanks to its implementation in 3D Slicer, a powerful tools for neuroimaging (Pieper et al. 2006).

\section{Methods and materials}

Implementation of GeodesicSlicer 


\section{Platform}

Geodesic Slicer was implemented in 3D Slicer (Pieper et al. 2006), a software which is freely downloadable from the website http://www.slicer.org. 3D Slicer provides an immense amount of functionality to visualize and analyze a wide range of datasets, such as anatomical/functional images, image segmentation results and surface models. Also, it supports import and export data from a wide range of standard data formats. In addition, the 3D Slicer has a widespread use in project research and is more and more downloaded (Pinter et al. 2012).

\section{Implementation}

The GeodesicSlicer module is written in Python. Python is a very popular and easily interpreted language, which allows multiple programming paradigms, including objectoriented, imperative and functional programming styles. Inheriting from 3D Slicer, GeodesicSlicer is available for Windows, Linux, and Mac OS X platforms.

Our implementation of the algorithm in 3D Slicer consists of a graphical user interface front-end to enable interactions of the user with the image and several algorithms back-end. It allows the generations of head surface mesh reconstruction and individualized 10-20 system EEG. Moreover, it can compute the geodesic distances between the target and electrodes landmarks and compute 2 correction factors to adjust rTMS dose for the treatment.

The geodesic distances (i.e. the shortest path between two points in a curved space) to draw the individualized 10-20 system EEG or compute the distances between the target and electrodes landmarks are calculated on a 3D mesh morphed to the structural MRI head data of the participant thanks to the implemented Dijkstra's algorithm (Dijkstra 1959), which calculates the shortest path between the vertex of triangle mesh.

Two correction factors to adjust rTMS dose for the treatment for individual subjects are given by the software. First, Stokes et al. (Stokes et al. 2007), proposed increasing the 
stimulation intensity by about $3 \%$ for each additional millimeter between the coil and the scalp surface. Second, Hoffman et al. (Ralph E. Hoffman et al. 2013) also take into account of the skin-surface-to-cortical-surface, but their adjustment also reflected the fact that magnetic field strength falls off exponentially relative to distance to the center of the coil (supplementary material of their article). These corrections factors are, for Stokes et al. (Stokes et al. 2007), where $[\operatorname{AdjMT} \%=2,7 *(\mathrm{SCDx}-\mathrm{SCDm})+\mathrm{rMT}]$ and according to Hoffman et colleagues (Ralph E. Hoffman et al. 2013), where [AdjMT\% = 0.90*rMT*e0.036*(SCDx-SCDm)], where AdjMT is the adjusted motor threshold in percent (\%), rMT is the unadjusted resting motor threshold in \% of stimulator output, SCDx is the scalp-to-cortex distance between the scalp and the cortical stimulation site and SCDm is the scalp-to-cortex distance between the scalp and the primary motor cortex (M1).

\section{Licensing and distribution}

GeodesicSlicer (WikiPage) is distributed under a CeCill license. The software may be used not only for research purposes but also in clinical and commercial projects. Note, however, that validation for a particular clinical purpose is an onus of the user. The brain stimulation guidelines and safety procedures are dependent on each neurostimulation therapy used [for example in TMS: (Rossi et al. 2009)], but not directly to the use of this software.

GeodesicSlicer modules can be downloaded as an extension for 3D Slicer 4.10.0 or higher. All the presented software is open-source and the source code is available on GitHub, which contains detailed guides for user's installation and usage. The authors declare that they have no conflict of interest.

\section{GeodesicSlicer, a MRI-guided method}

First, GeodesicSlicer provides realistic and accurate 3D representations of the head scalp. The, the MRI-guided method uses participant's individual MRI to determine the TMS coil position onto the head surface. We describe in the following section the workflow of 
GeodesicSlicer to position the TMS coil over the participant's head surface from her/his $\mathrm{T}_{1^{-}}$ weighted anatomical/functional images.

\section{Procedure of GeodesicSlicer}

1) Loading of the $T_{1}$-weighted image into $3 \mathrm{D}$ Slicer.

2) $3 \mathrm{D}$ representation of the head (the head surface mesh or more accurately, triangle meshes) was individually reconstructed in native space from the $\mathrm{T}_{1}$-weighted wholebrain anatomical image using 3D Slicer software ("editor toolbox" version 4.8).

3) Manual identification of four anatomical landmarks for the essential positioning of the electrodes on the head surface mesh: the nasion, the inion, the left and right tragi (in this order). The Dijkstra's algorithm automatically reconstructed the 10-20 system EEG with T3P3 in the middle of the segment delimited by T3 and P3. For that, the shortest paths between the nasion and inion and the left and right tragi that passed through the center point of the head (electrode $\mathrm{Cz}$ ). Then, always with the shortest path algorithm, all electrodes are located according to their standardized that represent proportions of the measured distance from the nasion to the inion and from the left to the right tragi (Klem et al. 1999).

4) Manual placement of the cortical stimulation target on the $T_{1}$-weighted image, the projection of it onto the head surface mesh was made by using a classical 3D Euclidean distance, i.e. $\sqrt{ }(\mathrm{x} 2-\mathrm{x} 1)^{2}+(\mathrm{y} 2-\mathrm{y} 1)^{2}+(\mathrm{z} 2-\mathrm{z} 1)^{2}$.

5) The fifth step was the computation of the geodesic distances between this projected target on the surface and the electrodes of the 10-20 system EEG corresponding of the participant's head. These three distances (in $\mathrm{cm}$ ) were then used to triangulate and to position the TMS coil manually over the participant's head.

6) The last step is needed to adjust the rTMS dose for treatment. After placing cortical landmark in M1 according to the Yousry's method (Yousry et al. 1997), the brain area 
to determine the motor threshold in rTMS, and giving rMT of stimulator output, the software gives two AdjMT of stimulator output.

The duration of this workflow lasted about 10 minutes per participant.

\section{Illustrative Examples Using GeodesicSlicer}

Use cases are presented to demonstrate the capabilities of GeodesicSlicer extension for addressing clinically relevant rTMS site determination. We chose brain imaging data of two patients from previous study of our team (Dollfus et al. 2018), but the rTMS stimulation proposed here is just theoretical. The subjects have previously written informed consent and these studies were approved by a local ethical committee. All coordinates are given in the MRI native space.

\section{Accuracy of the measure}

Eight controls ( $35.77 \pm 5.29$ years; 2 women) were included to assess the validity of the GeodesicSlicer method. The placement of the nasion, inion, and the two tragi determines the position of the electrodes in the 10-20 system EEG. We measured the distances from the nasion to the inion and from the left tragus and the right tragus in the controls with a measuring tape and compared them to the same distances calculated by GeodesicSlicer. Bland-Altman plots assess retest reliability of two measures and were used to test the stability across these distances (Bland and Altman 1999). 


\section{Results}

Illustrative Examples Using GeodesicSlicer

Case 1: Determination of the projected stimulation target in one patient with schizophrenia with auditory verbal hallucinations

The rTMS can be used as treatment for auditory verbal hallucinations (AVHs), notably, in the case of refractory to treatments (R. E. Hoffman et al. 1999). In the case described below, we will consider that patient receive a treatment by rTMS applied over a precise anatomical site in the left temporal region, that significant effects in AVH reduction.

The patient is a 35-year-old man, diagnosed with schizophrenia (based on the DSM-V, Diagnostic and Statistical Manual of Mental Disorder $5^{\text {th }}$ edition) and who suffers of constant AVHs. He was recruited from the University Hospital (Caen, France).

\section{rTMS site determination}

The patient underwent a structural MRI on a 3T scanner (Intera Achieva 3T, Philips Medical System, the Netherlands) with a three-dimensional (3D), high-resolution $\mathrm{T}_{1}$-weighted structural volume ( $T_{1}$ TFE sequence, $256 \times 256$ matrix size with 180 contiguous slices, field of view $(\mathrm{FOV})=256 \mathrm{~mm}, 1 \mathrm{~mm}$ isotropic resolution, antero-posterior slice orientation, repetition time $=6.914 \mathrm{~ms}$, echo time $=3.16 \mathrm{~ms}$, flip angle $=6$, inversion time $=940 \mathrm{~ms}$ ).

Using this system, the cortical stimulation target was localized at the crossing between the projection of the ascending branch of the left lateral sulcus and the left superior temporal sulcus. To do that, using a sagittal section of the structural MRI to visualize the upper sylvian and temporal sulcus, we propose to consider the intersection between the orthogonal projection of the verticalization of the Sylvius fissure and the upper left temporal sulcus (see Supplementary Data Video 1 in (Dollfus et al. 2018)).

GeodesicSlicer results 
After the different steps from GeodesicSlicer described above, the results are described below (see Fig. 1). The 10-20 system EEG electrodes were generated after determining the nasion $(x=-0.87, y=111.20, z=-22.57)$, the inion $(x=8.30, y=-101.80, z=-37.17)$, the left pre-auricular $(\mathrm{x}=-76.31, \mathrm{y}=7.92, \mathrm{z}=-42.99)$ and the right pre-auricular $(\mathrm{x}=4.30, \mathrm{y}=22.20$, $\mathrm{z}=-47.49)$ on the head surface mesh. After placing the cortical stimulation target on the patient's $T_{1}$ - weighted anatomical image $(x=-40.66, y=-23.26, z=3.57$, corresponding to the superior temporal sulcus), the stimulation target was projected on his head surface mesh. We then obtained the following 3 nearest electrodes around projected stimulation target and their geodesic distance (in $\mathrm{cm}$ ) with the projected stimulation target: Electrode 1: T3 at 3.65, electrode 2: T5 at 4.47 and electrode 3: $\mathrm{C} 3$ at 8.39 . These three distances were then used to triangulate and to position the TMS coil manually over the patient's head.

After placing M1 on the patient's $\mathrm{T}_{1}$ anatomical image $(\mathrm{x}=-30.92, \mathrm{y}=-11.77, \mathrm{z}=52.58)$ and choosing the stimulation intensity of the resting motor threshold (rMT) by default (100\%), we found the SCDx $=3.34 \mathrm{~cm}$, and $\mathrm{SCDm}=3.09 \mathrm{~cm}$ and the following two AdjMT (in \% stimulator): according to Strokes (Stokes et al. 2007): 107.22 and according to Hoffman (Ralph E. Hoffman et al. 2013): 98.75.

Case 2: Determination of the projected stimulation target in one patient with major depressive disorder

The rTMS can also be used as treatment for MDD that represent one of the most common psychiatric diseases with a prevalence in the general population general of $10-15 \%$. A large number of depressed patients are resistant to drug treatment and, actually, the rTMS is proving to be the greatest therapeutic efficacy (McGirr et al. 2015). In the case described below, we will consider that patient could, for example, receive a treatment by rTMS in stimulating a key region involved in the MDD: the left dorsolateral prefrontal (DLPFC) (McGirr et al. 2015). 
The patient is a 63-year-old woman, diagnosed with MDD (based on the DSM-V, Diagnostic and Statistical Manual of Mental Disorder $5^{\text {th }}$ edition). She was recruited from the University Hospital (Caen, France) and realized cerebral MRI on a 3T scanner in the same way that in the Case 1.

\section{rTMS site determination}

Using the structural MRI, after determining the position of the upper frontal sulcus and the lower frontal sulcus, the target can be defined as being equidistant from the upper and lower frontal sulcus in the coronal plane which crosses the anterior extremity of the temporal pole.

\section{GeodesicSlicer results}

The results from GeodesicSlicer are described below (see Fig. 2). The 10-20 system EEG electrodes were generated after determining the nasion $(x=-3.65, y=97.45, z=-5.77)$, the inion $(x=-3.14, y=-89.86, z=-16.90)$, the left pre-auricular $(x=-78.02, y=2.93, z=-$ 14.58) and the right pre-auricular $(x=71.20, y=2.88, z=-14.87)$ on the head surface of mesh. After placing the cortical stimulation target on the patient's $\mathrm{T}_{1}$-weighted anatomical image ( $\mathrm{x}$ $=-37.62, \mathrm{y}=40.13, \mathrm{z}=55.85$, corresponding to the patient's DLPFC), the stimulation target was projected on his head surface $(x=-54.22, y=49.50, z=66.96)$. Then, we obtained the following 3 nearest electrodes around projected stimulation target and their geodesic distance (in $\mathrm{cm}$ ) with the projected stimulation target: Electrode 1: F3 at 2.30, electrode 2: F7 at 3.73 and electrode 3: $\mathrm{C} 3$ at 4.03. With this these three distances, after triangulation, it could be possible to position the TMS coil manually over the patient's head.

After placing M1 area on the patient's $\mathrm{T}_{1}$ anatomical image $(\mathrm{x}=-32.49, \mathrm{y}=-3.51, \mathrm{z}=$ 71.66) and have choose the stimulation intensity of the rMT by default (100\%), we found SCDx $=22.06 \mathrm{~cm}$, and SCDm $=25.37 \mathrm{~cm}$ and the following two AdjMT\% (in \% stimulator): 
according to Strokes (Stokes et al. 2007): 90.73 and according to Hoffman (Ralph E. Hoffman et al. 2013): 79.88 .

\section{Accuracy of the measure}

The limits of agreement of Bland-Altman plots of the nasion-inion distances were from -0.82 to $1.96 \mathrm{~cm}$, with a mean difference of $0.57 \pm 0.70 \mathrm{~cm}$ (range, -0.64 to $1.59 \mathrm{~cm}$ ), and from -0.52 to $1.82 \mathrm{~cm}$ for the tragus-tragus with a mean difference of $0.65 \pm 0.59 \mathrm{~cm}$ (range, -0.16 to $1.53 \mathrm{~cm}$ ), showing that the reliability between the geodesic distances was consistent between those calculated by GeodesicSlicer and the manual measures. 


\section{Discussion}

Personalized and guided stimulation (Fox et al. 2013; I. E. Sommer et al. 2018) seems to be an efficient way to improve the discrepant efficacy results previously reported in NIBS studies (Briend et al. Under Review). In this context, we developed GeodesicSlicer, a novel MRI-guided method using individual brain imagery to position the TMS coil reliably on the participant's head. GeodesicSlicer aims to become a complete and easy-to-use toolkit for stimulation researchers by providing accurate target according to 10-20 system EEG electrode positioning and a correction factor to adjust the rTMS dose for the treatment.

In comparison with neuronavigation system, the current method may have some advantages because it is relatively inexpensive and does not require any additional experimental setting. In addition, the MRI-guided method could be particularly useful for therapeutic protocols, because the result of the software allows easy targeting of the same stimulation site across multiple sessions and in multicenter trials, as the three distances can be used offline to position the TMS coil (Andoh et al. 2009). Moreover, it only needs 3D Slicer, a software freely available online and is relatively user-friendly.

Previous studies have calculated geodesic distances between scalp landmarks using surface mesh representations of the head in order to guide NIBS (Andoh et al. 2009). Others have worked on the head surface mesh with vectors linking key anatomical landmarks drawn on the mesh and used it to calculate the precise distances on the scalp corresponding to these vectors (Vaghefi et al. 2015). Just one study has used a semi-automatic approach to generate the 10-20 system EEG correlates external skull locations (Xiao et al. 2017), but no study has combined triangulation system based on individualized 10-20 system EEG that morphed to the head surface mesh reconstruction of participant.

Others teams have developed a feasible low-cost solution to track coil positions during rTMS procedures, but the setup and run of the clinical experiment are time-consuming 
processes (Dayan et al. 2016; Rodseth et al. 2017; Washabaugh and Krishnan 2016). Although, our method that adjusts the 10-20 system EEG for the participant's skull size, conversely to other (Beam et al. 2009), have the benefit of taking into account differences in cortical anatomy or skull sizes between each individual in a quick and low-cost way. This triangulation-based MRI-guided method is an alternative to more complicated and costly stereotaxic targeting paradigms. In addition, this software package could be useful for laboratories that do not have access to neuronavigation system.

\section{Limitations and Future Works}

Our module has some limitations caused by the method itself. First, unlike probabilistic targeting techniques, Geodesic Slicer requires brain imaging. Indeed, MRI scanning is expensive and not always available in many institutes (Xiao et al. 2017), but this personalized method using MRI with the participant's own anatomical or functional images to guide is very accurate (Briend et al. Under Review; Kraus and Gharabaghi 2015; Lahti 2016; I. E. Sommer et al. 2018).

Second this method faces problems in time cost, mainly due to the manual positioning of the international 10-20 system EEG and the measurement procedure (Xiao et al. 2017). However by its facilitating approaches for rTMS target localization, the International 10-20 system of EEG represents the gold standard in clinical uses (Uwe Herwig et al. 2003).

Third, as Andoh and colleagues already mentioned (Andoh et al. 2009), the current MRI-guided method is not a neuronavigation system and therefore cannot provide online monitoring such as a real-time control of the coil angle. However, it is noteworthy that the influence of the coil angle remains debated (Niyazov et al. 2005).

Despite these limitations, there are several areas for future work. In particular, some steps of the MRI-guided method workflow under 3D Slicer can be automated as the landmarks 
placement including the nasion, the inion and the left and the right tragi. Moreover, the path precision calculated by the Dijkstra's algorithm produces “jagged" lines depends on the length of the triangle edges determined during the mesh creation. Further development of GeodesicSlicer should include these issues to resolve this possible variability, by adding a smoothing procedure that fitted the Dijkstra's algorithm path (Vaghefi et al. 2015).

Moreover, we did not assess the effectiveness of this costless method against neuronavigation on the one hand and on the other hand about the effectiveness of correction factors to adjust rTMS dose. The goal of this paper was to test the measurement accuracy of this software, but further studies with larger samples will be necessary to determine the clinical benefit of this method. Although, one paper that use triangulation-based MRI-guided method (Andoh et al. 2009) has already show the accuracy of it.

\section{Conclusions}

In summary, Geodesic Slicer is an alternative to the time-consuming process of neuronavigation system. The triangulation-based MRI-guided method presented herein provides a reproducible and inexpensive way to position the TMS coil that may be used in case of unavailability of online neuronavigation, for instance, in a clinical setting. This MRI-guided method can use cortical landmarks from all MRI scans. 
Figure with captions

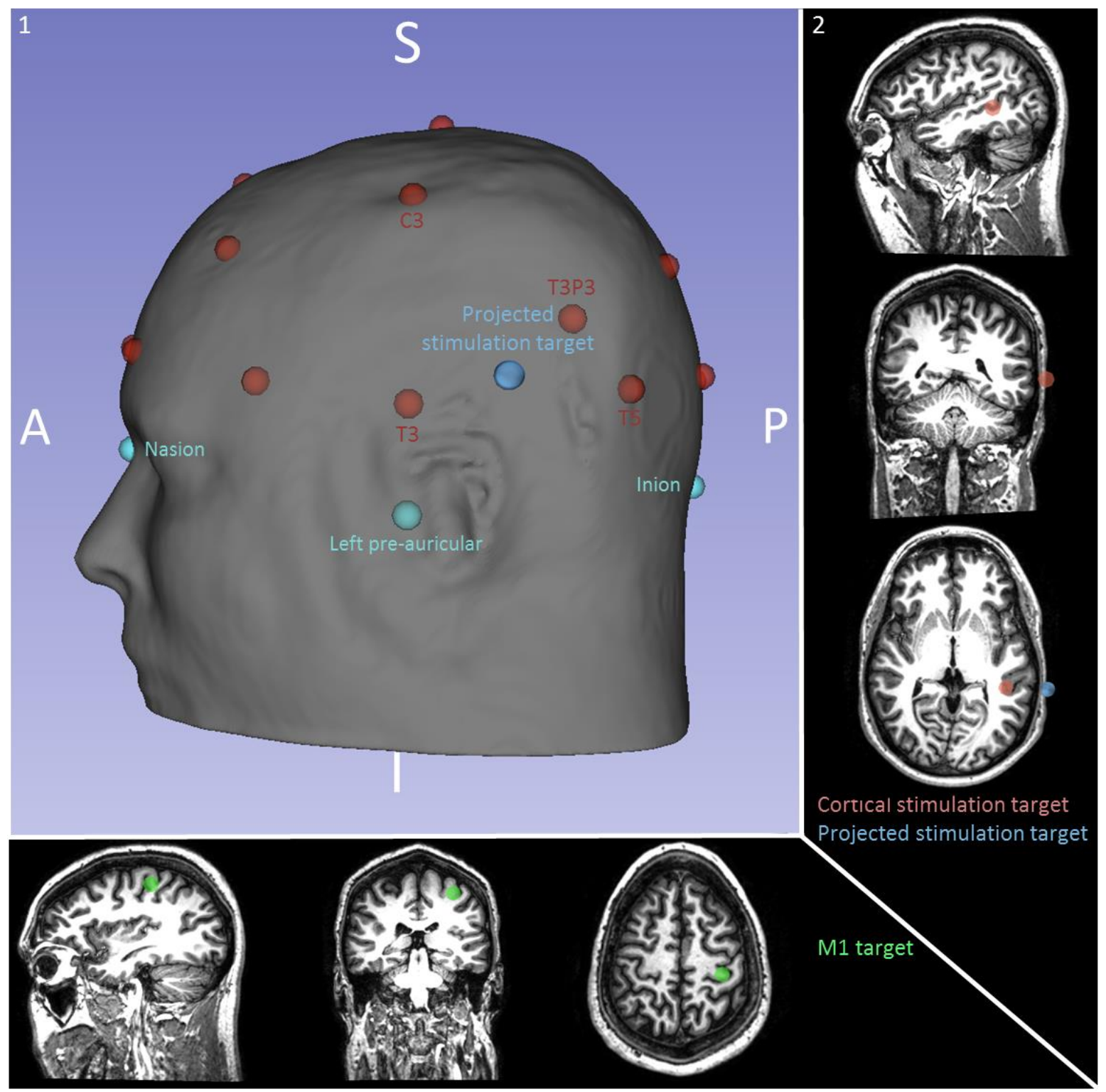

Fig. 1. Results of GeodesicSlicer in a patient with schizophrenia with auditory verbal hallucinations. 1. Representation of patient's head surface mesh generate by Geodesic Slicer with the nasion, inion and the left pre-auricular in turquoise, placed to generate the 10-20 system EEG in red. The stimulation target is then projected onto the head surface mesh (in blue) and localized near these three nearest electrodes with their geodesic distances: Here, the electrodes T3, T5 and C3. These three distances were then potentially used to triangulate and to position the TMS manually over the subject's head. 2. Views sagittal, coronal and axial of the cortical (in red) and projected (in blue) stimulation target. 3. Views sagittal, coronal and axial of the M1 area (in green). 


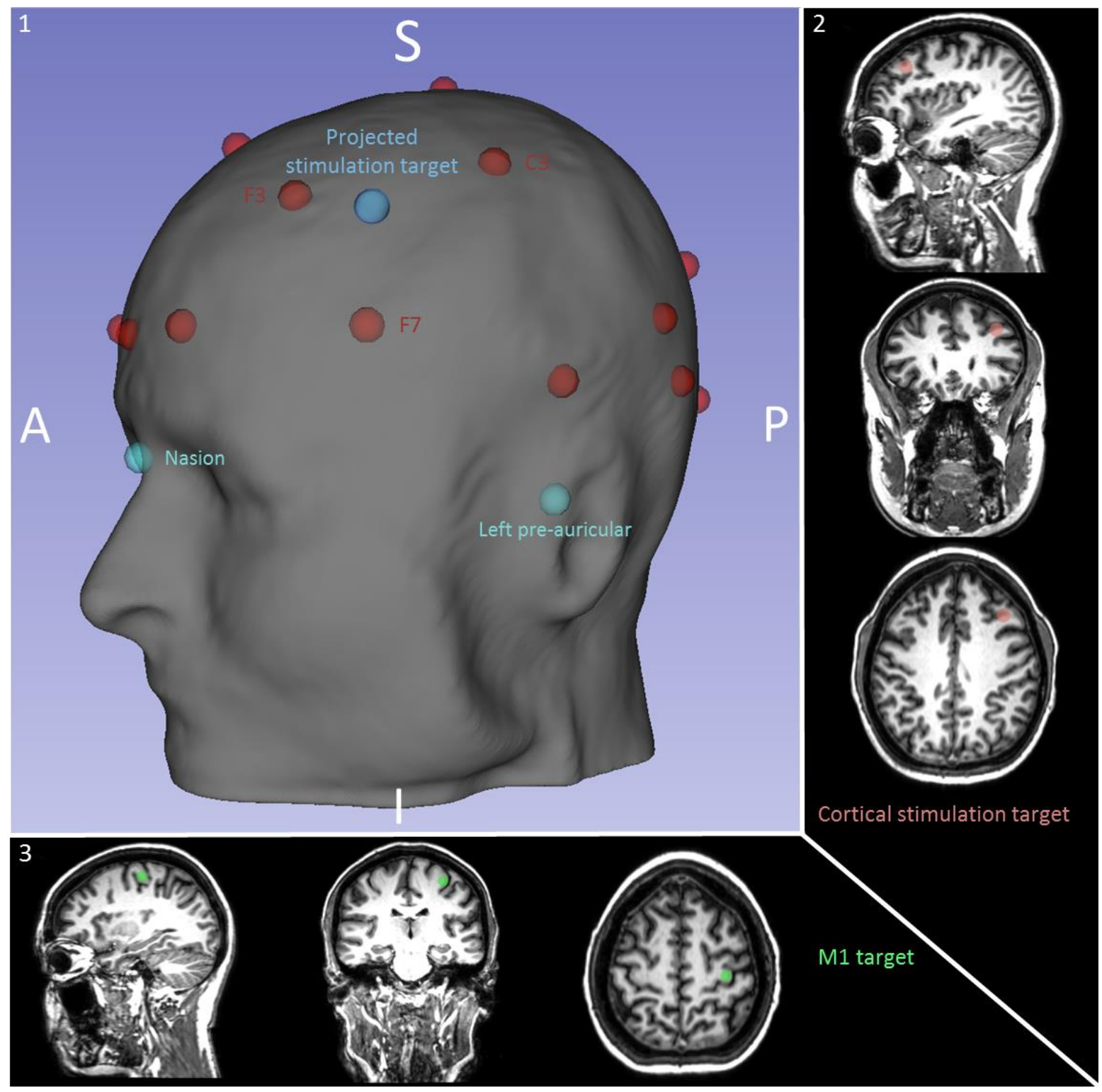

Fig. 2. Results of GeodesicSlicer in a patient with major depressive disorder. 1 . Representation of patient's head surface mesh generate by GeodesicSlicer with the nasion and the left pre-auricular in turquoise, notably placed to generate the 10-20 system EEG in red. The stimulation target is then projected onto the head surface mesh (in blue) and localized near these three nearest electrodes with their geodesic distances: Here, the electrodes F3, F7 and C3. In brain stimulation session, these three distances could be used to triangulate and to position the TMS manually over the patient's head. 2. Views sagittal, coronal and axial of the cortical stimulation target (in red). 3. Views sagittal, coronal and axial of the M1 area (in green). 
Information Sharing Statement: GeodesicSlicer has been put into a toolbox and can be download as an extension of $\underline{3 \mathrm{D} \text { Slicer }}$ or directly from Github.

The available implementation was uncoupled from the ethics protected image data used in the case studies. One brain imaging data example can be downloaded here. However, clinical data might be obtained upon request by contacting the corresponding author.

Acknowledgement: The authors would like to thank Drs A. Lasso and K. Yoshimi as well as A. Nourry for their valuable help in 3D Slicer and VTK library, and William P. Armstrong for the English rereading.

Conflict of interest: The authors have no conflict of interest to declare.

Funding source: This work was supported by the French Health Ministry (Programme Hospitalier de Recherche Clinique), the Fondation Fondamentale, the Association Perceneige, the Region Normandie and the University Caen Normandie. 


\section{References}

Andoh, J., Riviere, D., Mangin, J. F., Artiges, E., Cointepas, Y., Grevent, D., et al. (2009). A triangulation-based magnetic resonance image-guided method for transcranial magnetic stimulation coil positioning. Brain Stimul, 2(3), 123-31. https://doi.org/10.1016/j.brs.2008.10.002

Beam, W., Borckardt, J. J., Reeves, S. T., \& George, M. S. (2009). An efficient and accurate new method for locating the F3 position for prefrontal TMS applications. Brain stimulation, 2(1), 50-54. https://doi.org/10.1016/j.brs.2008.09.006

Bland, J. M., \& Altman, D. G. (1999). Measuring agreement in method comparison studies. Statistical Methods in Medical Research, 8(2), 135-160.

Briend, F., Nathou, C., Delcroix, N., Dollfus, S., \& Etard, O. (Under Review). A new toolbox to compare target localizations for non-invasive brain stimulation: An application of rTMS treatment for auditory hallucinations in schizophrenia. Schizophrenia Research.

Dayan, E., Thompson, R. M., Buch, E. R., \& Cohen, L. G. (2016). 3D-printed head models for navigated non-invasive brain stimulation. Clinical Neurophysiology: Official Journal of the International Federation of Clinical Neurophysiology, 127(10), 33413342. https://doi.org/10.1016/j.clinph.2016.08.011

De Witte, S., Klooster, D., Dedoncker, J., Duprat, R., Remue, J., \& Baeken, C. (2018). Left prefrontal neuronavigated electrode localization in tDCS: 10-20 EEG system versus MRI-guided neuronavigation. Psychiatry Research: Neuroimaging, 274, 1-6. https://doi.org/10.1016/j.pscychresns.2018.02.001

Dijkstra, E. W. (1959). A note on two problems in connexion with graphs. Numerische Mathematik, 1(1), 269-271. https://doi.org/10.1007/BF01386390

Dollfus, S., Jaafari, N., Guillin, O., Trojak, B., Plaze, M., Saba, G., et al. (2018). HighFrequency Neuronavigated rTMS in Auditory Verbal Hallucinations: A Pilot Double- 
Blind Controlled Study in Patients With Schizophrenia. Schizophrenia Bulletin, 44(3), 505-514. https://doi.org/10.1093/schbul/sbx127

Fox, M. D., Liu, H., \& Pascual-Leone, A. (2013). Identification of reproducible individualized targets for treatment of depression with TMS based on intrinsic connectivity. NeuroImage, 66, 151-160. https://doi.org/10.1016/j.neuroimage.2012.10.082

Herbsman, T., \& Nahas, Z. (2011). Anatomically based targeting of prefrontal cortex for rTMS. Brain Stimulation, 4(4), 300-302. https://doi.org/10.1016/j.brs.2011.01.004

Herwig, U., Padberg, F., Unger, J., Spitzer, M., \& Schönfeldt-Lecuona, C. (2001). Transcranial magnetic stimulation in therapy studies: examination of the reliability of “standard" coil positioning by neuronavigation. Biological Psychiatry, 50(1), 58-61.

Herwig, Uwe, Satrapi, P., \& Schönfeldt-Lecuona, C. (2003). Using the international 10-20 EEG system for positioning of transcranial magnetic stimulation. Brain Topography, 16(2), 95-99.

Hoffman, R. E., Boutros, N. N., Berman, R. M., Roessler, E., Belger, A., Krystal, J. H., \& Charney, D. S. (1999). Transcranial magnetic stimulation of left temporoparietal cortex in three patients reporting hallucinated "voices." Biological Psychiatry, 46(1), $130-132$.

Hoffman, Ralph E., Wu, K., Pittman, B., Cahill, J. D., Hawkins, K. A., Fernandez, T., \& Hannestad, J. (2013). Transcranial magnetic stimulation of Wernicke's and right homologous sites to curtail "voices:" a randomized trial. Biological psychiatry, 73(10), 1008-1014. https://doi.org/10.1016/j.biopsych.2013.01.016

Jasper, H. (1958). The ten twenty electrode system of the international federation. Electroencephalography and Clinical Neurophysiology, 10, 371-375. 
Klem, G. H., Lüders, H. O., Jasper, H. H., \& Elger, C. (1999). The ten-twenty electrode system of the International Federation. The International Federation of Clinical Neurophysiology. Electroencephalography and Clinical Neurophysiology. Supplement, 52, 3-6.

Kraus, D., \& Gharabaghi, A. (2015). Projecting Navigated TMS Sites on the Gyral Anatomy Decreases Inter-subject Variability of Cortical Motor Maps. Brain Stimulation, 8(4), 831-837. https://doi.org/10.1016/j.brs.2015.03.006

Lahti, A. C. (2016). Making Progress Toward Individualized Medicine in the Treatment of Psychosis. The American Journal of Psychiatry, 173(1), 5-7. https://doi.org/10.1176/appi.ajp.2016.15101320

McGirr, A., Van den Eynde, F., Tovar-Perdomo, S., Fleck, M. P. A., \& Berlim, M. T. (2015). Effectiveness and acceptability of accelerated repetitive transcranial magnetic stimulation (rTMS) for treatment-resistant major depressive disorder: an open label trial. Journal of Affective Disorders, 173, 216-220. https://doi.org/10.1016/j.jad.2014.10.068

Niyazov, D. M., Butler, A. J., Kadah, Y. M., Epstein, C. M., \& Hu, X. P. (2005). Functional magnetic resonance imaging and transcranial magnetic stimulation: Effects of motor imagery, movement and coil orientation. Clinical Neurophysiology, 116(7), 16011610. https://doi.org/10.1016/j.clinph.2005.02.028

Pieper, S., Lorensen, B., Schroeder, W., \& Kikinis, R. (2006). The NA-MIC Kit: ITK, VTK, pipelines, grids and 3D slicer as an open platform for the medical image computing community. In 3rd IEEE International Symposium on Biomedical Imaging: Nano to Macro, 2006. (pp. 698-701). Presented at the 3rd IEEE International Symposium on Biomedical Imaging: Nano to Macro, 2006. https://doi.org/10.1109/ISBI.2006.1625012 
Pinter, C., Lasso, A., Wang, A., Jaffray, D., \& Fichtinger, G. (2012). SlicerRT: radiation therapy research toolkit for 3D Slicer. Medical Physics, 39(10), 6332-6338. https://doi.org/10.1118/1.4754659

Rodseth, J., WashaBaugh, E. P., \& Krishnan, C. (2017). A Novel Low-Cost Approach for Navigated Transcranial Magnetic Stimulation. Restorative neurology and neuroscience, 35(6), 601-609. https://doi.org/10.3233/RNN-170751

Rossi, S., Hallett, M., Rossini, P. M., Pascual-Leone, A., \& Safety of TMS Consensus Group. (2009). Safety, ethical considerations, and application guidelines for the use of transcranial magnetic stimulation in clinical practice and research. Clinical Neurophysiology: Official Journal of the International Federation of Clinical Neurophysiology, 120(12), 2008-2039. https://doi.org/10.1016/j.clinph.2009.08.016

Sommer, I. E. C., de Weijer, A. D., Daalman, K., Neggers, S. F., Somers, M., Kahn, R. S., et al. (2007). Can fMRI-guidance improve the efficacy of rTMS treatment for auditory verbal hallucinations? Schizophrenia Research, 93(1-3), 406-408. https://doi.org/10.1016/j.schres.2007.03.020

Sommer, I. E., Kleijer, H., \& Hugdahl, K. (2018). Toward personalized treatment of hallucinations. Current Opinion in Psychiatry, 31(3), 237-245. https://doi.org/10.1097/YCO.0000000000000416

Stokes, M. G., Chambers, C. D., Gould, I. C., English, T., McNaught, E., McDonald, O., \& Mattingley, J. B. (2007). Distance-adjusted motor threshold for transcranial magnetic stimulation. Clinical Neurophysiology, 118(7), 1617-1625. https://doi.org/10.1016/j.clinph.2007.04.004

Summers, P. M., \& Hanlon, C. A. (2017). BrainRuler-a free, open-access tool for calculating scalp to cortex distance. Brain stimulation, 10(5), 1009-1010. https://doi.org/10.1016/j.brs.2017.03.003 
Trojak, B., Meille, V., Chauvet-Gelinier, J.-C., \& Bonin, B. (2012). Does the intensity of transcranial magnetic stimulation need to be adjusted to scalp-cortex distance? The Journal of Neuropsychiatry and Clinical Neurosciences, 24(2), E13. https://doi.org/10.1176/appi.neuropsych.11050114

Vaghefi, E., Cai, P., Fang, F., Byblow, W. D., Stinear, C. M., \& Thompson, B. (2015). MRI Guided Brain Stimulation without the Use of a Neuronavigation System. BioMed Research International, 2015, 647510. https://doi.org/10.1155/2015/647510

Washabaugh, E. P., \& Krishnan, C. (2016). A low-cost system for coil tracking during transcranial magnetic stimulation. Restorative Neurology and Neuroscience, 34(2), 337-346. https://doi.org/10.3233/RNN-150609

Xiao, X., Zhu, H., Liu, W.-J., Yu, X.-T., Duan, L., Li, Z., \& Zhu, C.-Z. (2017). Semiautomatic 10/20 Identification Method for MRI-Free Probe Placement in Transcranial Brain Mapping Techniques. Frontiers in Neuroscience, 11, 4. https://doi.org/10.3389/fnins.2017.00004

Yousry, T. A., Schmid, U. D., Alkadhi, H., Schmidt, D., Peraud, A., Buettner, A., \& Winkler, P. (1997). Localization of the motor hand area to a knob on the precentral gyrus. A new landmark. Brain: A Journal of Neurology, 120 ( Pt 1), 141-157. 